\title{
Hypoglycaemic effect of coixan from Coix seeds on mice
}

\author{
Yanan Yin ${ }^{1,2,3}$, Weiwei Yang ${ }^{1,2,3}$, Yingliang Jiao ${ }^{1,2,3}$, He Yang ${ }^{1,2,3}$, Xinglin $\mathrm{Li}^{1,2,3, *}$ \\ ${ }^{1}$ State Key Laboratory of Food Nutrition and Safety, Tianjin, 300457, China \\ ${ }^{2}$ National Demonstration Center for Experimental Bioengineering Education(Tianjin University of Science and Technology), Tianjin, \\ 300457, China \\ ${ }^{3}$ College of Bioengineering, Tianjin University of Science and Technology, Tianjin, 300457, China
}

\begin{abstract}
Diabetes is an endocrine system disease characterized by high morbidity, high prevalence, and high mortality. Although the traditional medicine for treating diabetes has a good effect, its side effects are also large. Traditional Chinese medicine has also achieved good therapeutic effects in the treatment of diabetes, and the side effects of Chinese medicine are small. Coix is a medicinal and edible plant. It is used as food and medicine alone or in combination with other herbs in the early days. Its seeds have become one of the most popular Chinese herbal medicines. The coixan was extracted from Coix seed, and it was administered to diabetic mice. After five weeks of treatment, it was found to have some role in hypoglycemic. This study provides a good application prospect for Chinese medicine treatment of diabetes.
\end{abstract}

\section{Introduction}

As a very common chronic disease, diabetes has become the third biggest killer of human health due to its high prevalence, high morbidity and high mortality. Diabetic patients cannot produce or use insulin properly, so their blood glucose levels are high. Diabetes is divided into two types, type I and type II. Type I diabetes is insulin-dependent diabetes mellitus, which does not produce insulin in the body and requires daily insulin injection to maintain normal blood glucose levels. Type II diabetes is non-insulin-dependent diabetes, the body can't produce enough insulin, or cannot use insulin correctly, is the most common diabetes ${ }^{1}$. Although traditional drugs for treating diabetes have good hypoglycemic effects, their side effects are also very large. Traditional Chinese medicine has achieved good clinical practice in the treatment of diabetes, and its side effects are small, so it has broad application prospects. Coix is an annual or perennial herb that has been used as a food and medicine alone or in combination with other herbs since ancient times. Its seeds have become one of the most popular Chinese herbal medicines, containing protein, lipids, carbohydrates, crude fiber, calcium, phosphorus, iron and other ingredients ${ }^{2,3}$. It has been found to have biological activities such as anti-oxidation, anti-inflammatory, immune regulation, anti-cancer, anti-obesity, anti-diabetes and blood lipid regulation ${ }^{2,4-6}$. This paper mainly introduces the study of in vivo anti-hyperglycemia of coixan extracted from Coix seed, which proves that the coixan extracted by this method has the effect of decrease blood glucose levels.

\subsection{Materials}

Coix powder: Coix seed from Chifeng, Inner Mongolia Autonomous Region, used after cyclone grinding and powdering.

Experimental animal: healthy male Kunming mice with specific pathogens free (SPF) grade provided by Beijing Weitong Lihua Experimental Animal Technology Co., Ltd.

Experimental animal diet: normal diet is $100 \%$ basal chow, high-carbonhydrate diet is $53.7 \%$ basal chow, and $20 \%$ sucrose, $15 \%$ lard, $10 \%$ egg yolk powder, $1.1 \%$ cholesterol, $0.2 \%$ sodium cholate.

\subsection{Experimental methods}

\subsubsection{Extraction procedure}

First, Coix powder was degreased with n-hexane with a Soxhlet extractor. The degreased Coix powder was added to distilled water, heated in a water bath at $80^{\circ} \mathrm{C}$ for $3 \mathrm{~h}$, and the supernatant was centrifuged. After concentrated, $\alpha$-amylase was added, and heated in a water bath at $55-60^{\circ} \mathrm{C}$ for $1 \mathrm{~h}$, followed by addition of saccharifying enzyme at $55-60^{\circ} \mathrm{C}$ water bath heated again $1 \mathrm{~h}$. Then we used savage reagent to deproteinize, discard protein after adding alcohol, overnight, centrifuged to take the precipitate, added the precipitate to distilled water to dissolve, discarded the insoluble part, put the obtained solution into the $-80^{\circ} \mathrm{C}$ refrigerator for $24 \mathrm{~h}$, and prepared Freeze-dried. The lyophilized powder is coixan we prepared.

\section{Materials and methods}

\footnotetext{
*Correspondence author, Email: 1xlszf@tust.edu.cn
} 


\subsubsection{Experimental animal feeding environment}

Mice were housed in a clean, well ventilated rat room at room temperature $25 \pm 1^{\circ} \mathrm{Cand}$ relative humidity $60 \pm$ $5 \%$. This animal study was approved by the Institutional Animal Care and Use Committee of China, and institutional guidelines for animal welfare and experimental conduct were followed.

\subsubsection{Making model of diabetic mice and experimental scheme}

The mice were adaptively fed after one week, the mice prepared for modeling were fed a high-carbonhydrate diet, and the control mice were fed a normal diet. Three weeks after the feeding, the diabetes model was made using streptozotocin (STZ) at a dose of $100 \mathrm{mg} / \mathrm{kg}$ for the fourth week. One week later, the fasting blood glucose was measured, and the fasting blood glucose was greater than $11.1 \mathrm{mM}$ as a standard to judge whether the modeling was successful. The unsuccessful mice model of diabetes was re-injected with STZ at a dose of 60 $\mathrm{mg} / \mathrm{kg}$. One week later, the fasting blood glucose was measured and grouped into normal diet group (represented by $\mathrm{Nd}$ ), high-carbonhydrate diet group (represented by $\mathrm{Hc}$ ), the coixan was treated with normal diet group (represented by $\mathrm{Nd}-\mathrm{Cn}$ ), the coixan was treated with high-carbonhydrate diet group (represented by $\mathrm{Hc}-\mathrm{Cn}$ ), and the positive drug was treated with high-carbonhydrate diet group (represented by Hc-Pd).

After grouping is completed, the $\mathrm{Nd}$ group and $\mathrm{Hc}$ group were intragastrically administered with normal saline every day. The Nd-Cn group and $\mathrm{Hc}-\mathrm{Cn}$ group were intraperitoneally injected with coixan (dissolved with physiological saline) at a dose of $100 \mathrm{mg} / \mathrm{kg}$ per day, and the Hc-Pd group was intragastrically administered daily with acarbose at a dose of $100 \mathrm{mg} / \mathrm{kg}$. The dosing period was 35 days. The body weight, food consumption, water consumption, fasting blood glucose, urine glucose, and blood glucose were measured weekly during the study.

\section{Results}

After 5 weeks of administration, the body weight before and after the administration, and the changes in the food consumption and water consumption after administration carry out significant analysis. Compared with the $\mathrm{Nd}$ group, there was no significant change in the $\mathrm{Nd}-\mathrm{Cn}$ group, the Hc group decreased significantly, and the Hc-Pd group and Hc-Cn group had decreased very significantly at the fifth week weight body that was not administered; there was no significant change in the body weight of the mice after administration, although the $\mathrm{Hc}-\mathrm{Pd}$ group and $\mathrm{Hc}-\mathrm{Cn}$ group were still reduced compared with the $\mathrm{Nd}$ group, but there was no significant difference, and the Hc group was significantly decreased; there was no significant change in the Nd-Cn group, $\mathrm{Hc}$ group, and Hc-Cn group in the dietary changes of each group after administration, and the increase in the $\mathrm{Hc}-\mathrm{Pd}$ group was extremely significant; there was no significant change in the Nd-Cn group after the administration of drinking water, and the water intake is increased in the $\mathrm{Hc}$ group and $\mathrm{Hc}-\mathrm{Cn}$ group that was extremely significant, while the Hc-Pd group was significantly increased. Compared with the Hc group, the $\mathrm{Nd}$ group and $\mathrm{Nd}-\mathrm{Cn}$ group increased significantly, while the $\mathrm{Hc}-\mathrm{Pd}$ group and $\mathrm{Hc}-\mathrm{Cn}$ group did not change significantly at the fifth week of body weight; for the body weight of mice at the tenth week, the significant changes in each group compared with the Hc group were consistent with the changes at the fifth week of body weight; there was no significant difference in the diet of each group after administration compared with the $\mathrm{Hc}$ group; the change of drinking water after administration was reduced in each group compared with the Hc group, and it was extremely significant (Fig. 1).
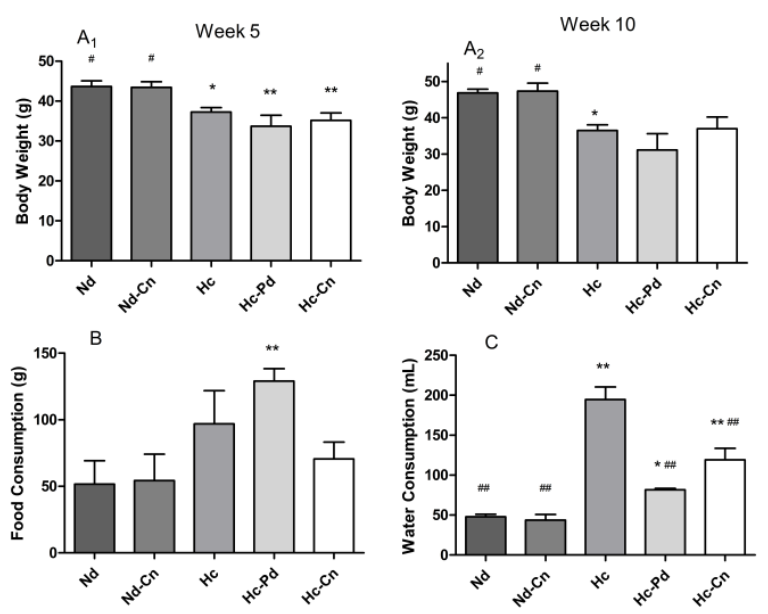

Fig.1 - Effect of Coixan on general health condition of the diabetic mice. (A) Body weight changes. A1, Data at the 5th week. A2, Data at the 10 th week. (B) The average food consumption. (C) The average water consumption. ${ }^{*} p<0.05$, $*_{*} \mathrm{p}<0.01$, significant differences between two groups and all compared to normal group. $\# \mathrm{p}<0.05$, \#\#p $<0.01$, significant differences between two groups and all compared to model group. $\mathrm{Nd}$, normal diet group. $\mathrm{Nd}-\mathrm{Cn}$, the coixan was treated with normal diet group. Hc, high-carbonhydrate diet group. $\mathrm{Hc}-\mathrm{Pd}$, the positive drug was treated with high-carbonhydrate diet group. Hc-Cn, the coixan was treated with high-carbonhydrate diet group.

The fasting blood glucose was measured at the fifth week before the administration, compared with the $\mathrm{Nd}$ group, the Hc-Pd group was high and it was extremely significant, while the $\mathrm{Hc}-\mathrm{Cn}$ group was significantly increased, although the fasting blood glucose also increased in the Hc group, but there was no significant change in the $\mathrm{Nd}$ group, and there was no significant change in the Nd-Cn group; there was no significant change in each group compared to the Hc group. The fasting blood glucose of each group was measured at the tenth week after administration, compared with the $\mathrm{Nd}$ group, the increase in the Hc group and Hc-Cn group was extremely significant, the Hc-Pd group was significantly increased, and the Nd-Cn group was not significantly changed; compared with the Hc group, the Nd group and Nd-Cn group showed a decrease and it was extremely significant, while the Hc-Pd group and $\mathrm{Hc}-\mathrm{Cn}$ group showed no significant change (Fig. 2A). 
At the fifth week, the urine glucose was measured, and compared with the $\mathrm{Nd}$ group, each group increased, and it was extremely significant; compared with the Hc group, the $\mathrm{Nd}$ group and $\mathrm{Nd}-\mathrm{Cn}$ group decreased, and it was extremely significant, and there were no significant difference in other groups. At the tenth week after administration, compared with the $\mathrm{Nd}$ group, except for the Nd-Cn group had no significant difference, and the other groups were extremely significantly increased; compared with the Hc group, there was no significant difference between the Hc-Cn group and Hc-Pd group, and the other two groups were reduced, and they were most significant (Fig. 2B).

At the tenth week, the oral glucose tolerance test was measured after administration. At 0 min, compared with the $\mathrm{Nd}$ group, there was no significant change in the $\mathrm{Nd}-\mathrm{Cn}$ group, and the Hc group and the Hc-Cn group were increased and they were extremely significant, and the Hc-Pd group was significantly increased; compared with the Hc group, there was no significant change in the Hc-Pd group and Hc-Cn group, and the other two groups showed a decrease and it was extremely significant. At $30 \mathrm{~min}$, the significant change trend was consistent with 0 min compared with the $\mathrm{Nd}$ group; compared with the Hc group, the significant change was still the same as the trend at $0 \mathrm{~min}$. At $60 \mathrm{~min}$, compared with the $\mathrm{Nd}$ group, there was no significant change in the Nd-Cn group and Hc-Cn group, and the Hc group and Hc-Pd group showed extremely significant increase; compared with Hc group, there were no significant changes in the Hc-Cn group and Hc-Pd group, and the other two groups were significantly less. At $90 \mathrm{~min}$, compared with the $\mathrm{Nd}$ group, the $\mathrm{Hc}$ group, the Hc-Pd group, and the Hc-Cn group showed an increasing trend, and it was extremely significant, and the other groups had no significant change; compared with the Hc group, the $\mathrm{Nd}-\mathrm{Cn}$ group and $\mathrm{Nd}$ group were extremely significantly decreased, the Hc-Pd group decreased significantly, and the Hc-Cn group showed no significant change. At $120 \mathrm{~min}$, compared with the $\mathrm{Nd}$ group, there was no significant change in the other groups except for the extremely significant increase in the Hc group; compared with the Hc group, the Nd group and $\mathrm{Nd}-\mathrm{Cn}$ group decreased, and it was extremely significant, although the Hc-Pd group and the $\mathrm{Hc}-\mathrm{Cn}$ group were lower than the Hc group, there was no significant difference with the Hc group (Fig. 2C).
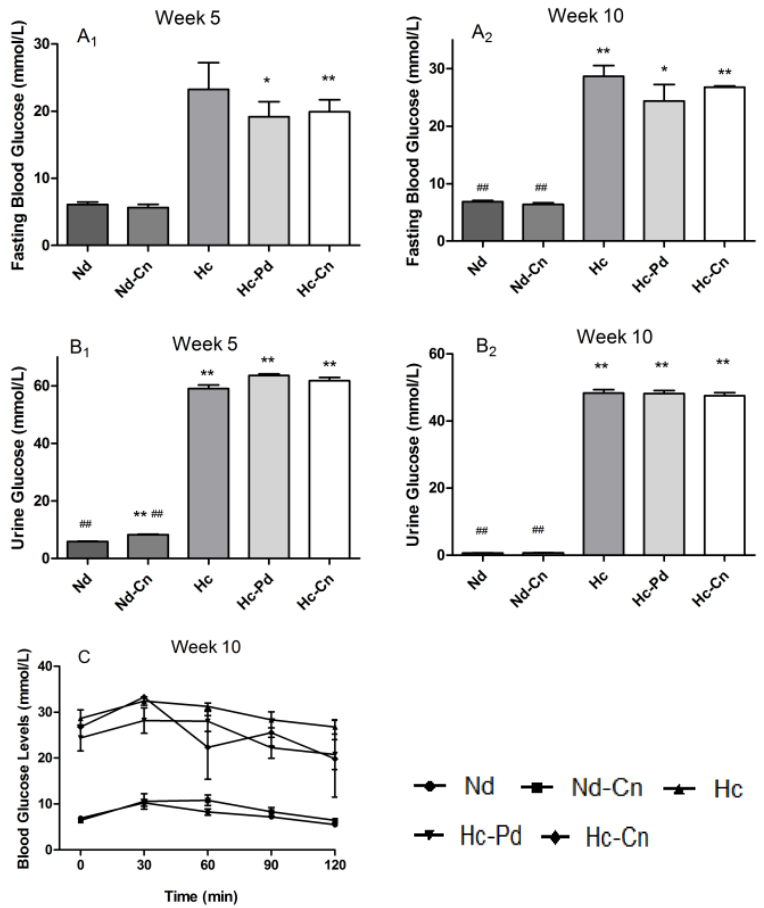

Fig.2 - Blood glucose responses in STZ-treated mices. (A) FBG detection. A1, Data at the 5th week. A2, Data at the 10th week. (B) Urinary glucose measurement. B1, Data at the 5th week. B2, Data at the 10th week. (C) Oral glucose tolerance test (OGTT). ${ }^{*} \mathrm{p}<0.05,{ }^{*} \mathrm{p}<0.01$, significant differences between two groups and all compared to normal group. $\# \mathrm{p}<0.05, \# \# \mathrm{p}<0.01$, significant differences between two groups and all compared to model group. $\mathrm{Nd}$, normal diet group. $\mathrm{Nd}-\mathrm{Cn}$, the coixan was treated with normal diet group. Hc, high-carbonhydrate diet group. Hc-Pd, the positive drug was treated with high-carbonhydrate diet group. Hc-Cn, the coixan was treated with high-carbonhydrate diet group.

\section{Conclusion}

According to the changes of body weight, food consumption, water consumption before and after treatment, diabetic mice were characterized by polydipsia, polyphagia and weight loss. According to the changes of the fasting blood glucose in diabetic mice before and after treatment, it was concluded that the fasting blood glucose of each group gradually increased with time, while coixan did not significantly reduce fasting blood glucose, but it was lower than the model group. From the oral glucose tolerance test measured in the tenth week, it can be inferred that coixan has a certain effect on reducing postprandial blood glucose. Although there was no significant difference compared with the model group, but there had been a significant decrease trend. The glucose content in urine of diabetic mice increased, and coixan had no significant effect on the urine glucose of normal mice and diabetic mice. Through this experiment, we found that coixan has a certain therapeutic effect on diabetes. This also provides a good application prospect for Chinese medicine treatment of diabetes. 


\section{Acknowledgement}

This work was supported by National Natural Science Foundation of China-Huge Scientific Equipment Scientific Research of China Academy of Sciences (No. U1332123).

\section{References}

1. W. L. Li, H. C. Zheng, J. Bukuru \& N. K. De. Natural medicines used in the traditional Chinese medical system for therapy of diabetes mellitus. Journal of Ethnopharmacology 92, 1-21 (2004).

2. F. Yu et al. Research and Application of Adlay in Medicinal Field. Chinese Herbal Medicines 9, 126-133 (2017).

3. F. Zhu. Coix: Chemical composition and health effects. Trends in Food Science \& Technology 61, 160-175 (2017).

4. S. O. Kim et al. Hypolipidemic effects of crude extract of adlay seed (Coix lachrymajobi var. mayuen) in obesity rat fed high fat diet: relations of TNF-alpha and leptin mRNA expressions and serum lipid levels. Life Sciences 75, 1391-1404 (2004).

5. X. Lu et al. A polysaccharide fraction of adlay seed ( Coix lachryma - jobi L.) induces apoptosis in human non-small cell lung cancer A549 cells. Biochemical \& Biophysical Research Communications 430, 846-851 (2013).

6. R. M. Perez G, M. A. Zavala S, G. S. Perez \& G. C. Perez. Antidiabetic effect of compounds isolated from plants. Phytomedicine 5, 55-75 (1998). 